\title{
Gamma irradiation in modulating cadmium bioremediation potential of Aspergillus sp.
}

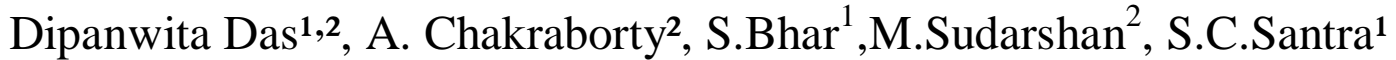 \\ ${ }^{1}$ Department of Environmental Science, University of Kalyani, Kalyani,Nadia 741235, W.B, India \\ ${ }^{2} U G C-D A E$ Consortium for Scientific Research, Kolkata Centre,3/LB-8,Saltlake, Kolkata 700098,India
}

\begin{abstract}
The present paper describes the potential of gamma irradiation in modulating Cd tolerance of Aspergillus sp. After being exposed to different gamma absorbed doses (20-100Gy), Aspergillus sp. grown in different $C d$ supplemented media, showed increase in growth (expressed in terms of colony forming unit), higher efficiency in Cd accumulation and removal, when compared to that of their unirradiated counterparts. Our results throw light towards a new step of Cd bioremediation.
\end{abstract}

Key words: Aspergillus sp, Bioremediation, Cadmium, Gamma irradiation

\section{INTRODUCTION}

In recent years increase in pollution load from industrial effluents has become a serious global issue. Heavy metals constitute a major component of such unwanted industrial effluents. Pollution caused by these heavy metals has created an alarming situation as they persist for very long time in the abiotic components of the ecosystem and are discharged into thereby creating severe hazards to the various strata of living world. In most of the countries $\mathrm{Cd}, \mathrm{Hg}, \mathrm{Pb}$ are included among the 'priority pollutants' because of their high toxicity.

For controlling such heavy metal pollution different strategies are being planned and implemented, but most of these processes are not only costly but also less effective. As such abatement of heavy metal pollution through bioremediation has gained a major focus and is being regarded as an cost-effective alternative solution. Metal accumulation potential of different microorganisms like bacteria, fungi, algae has placed them to be considered as effective agents for bioremediation of heavy metals. Among these microbes, fungi have better prospective to be used in bioremediation as they can accumulate more metal due to their high surface to volume ratio than the other microbes. Potential of filamentous fungi in bioremediation of heavy metals containing industrial effluents and waste waters has been reported from different parts of the world [1,2,3] .Among different filamentous fungi Aspergillus niger has been consistently listed among the top metal biosorbents [1,47]. Actually research in mycology has taken a different term due to the natural inherent potential of various fungi in having productive values which are highly beneficial for human welfare. While some fungi produce enzymes that are required for industrial purposes [8], while some are effective in waste detoxification [9-10]. To have more such economic output, recently research and developmental activities in terms of genetic engineering is being used for microbial strain improvement. Studies are also being carried out to improve the strains so as to make them more efficient accumulators of metals. Among different strain improving agent physical mutagen like ionizing radiation treatment deserves mention. Geweely and Nawar [11] reported that low doses of gamma causes stimulatory effects in fungi like enhancement in spore germination percentage, mycelial growth ; while higher doses causes killing of those fungi. Aspergillus niger, Rhizopus microsporus and Penicillium atrovenetum showed enhance production of industrially important enzyme lipase when these were exposed to various low doses of gamma irradiation (20, 40, 60, 80, 100, 120, 140 and $160 \mathrm{~Gy})[12]$.

The present study is designed to observe the potential of gamma in modulating Cd tolerance of Aspergillus sp grown in Cd stress.

\subsection{Isolation, Identification and Culture of fungi :}

\section{MATERIALS AND METHODS}

Aspergillus sp. (black conidial heads) was isolated from soil of garbage dump site of Dhapa, Kolkata by standard plating methods [13] in Potato dextrose media. The species was purified by streaking repeatedly on the same medium and the fungus was identified by high resolution microscopy.

Fungal strain was cultured in potato dextrose broth with the following composition: Peeled potato (400gm), Dextrose $(25 \mathrm{gm})$, dissolved in one liter of distilled water. Final $\mathrm{pH}$ was around 7 . The medium was autoclaved at $121^{\circ} \mathrm{C}$ for 20 minutes. Cultures were maintained in agar slants (potato dextrose broth plus $20 \mathrm{~g} / \mathrm{l}$ agar). 


\subsection{Assessment of metal (Cd) tolerance of isolated Apergillus sp.:}

Assessment of metal (Cd) tolerance was done in two steps. Initially, determination of Minimum Inhibitory Concentration (MIC) of Aspergillus sp.(isolated from soil near garbage dump site of Dhapa, Kolkata ) against $\mathrm{Cd}$ was done. Different concentrations of metal solutions were added separately to PDA medium and being inoculated with $100 \mu \mathrm{l}$ of spore suspension to determine MIC (minimum inhibitory concentration). $\mathrm{CdCl}_{2}, \mathrm{H}_{2} \mathrm{O}$ (Merck) salt was used for running all experiments. Secondly, further evaluation of Cd tolerance after being exposed to different gamma absorbed doses were studied considering the changes in number of colonies (in terms of colony forming unit; $\mathrm{CFU}$ ) between gamma exposure and non-exposure groups. For these, preparation of spore suspension and gamma exposure to that spore suspensions were necessary, The steps are briefly stated below.

\subsubsection{Preparation of Spore suspension:}

8days old culture (grown in potato dextrose broth media) was agitated fully and then hyphal mat was removed and the liquid was filtered through a Whatmann filter paper No-1.Filtrate was centrifuged in 10,000 $\mathrm{rpm}$ for $15 \mathrm{mins}$, supernatant was discarded and the pellet was suspended in Tween $20(0.02 \% \mathrm{v} / \mathrm{v})$ and $\mathrm{NaCl}$ $(0.85 \% \mathrm{w} / \mathrm{v})$ solution [14-15].

\subsubsection{Gamma radiation Exposure to spore suspension :}

Spore suspensions ( $5 \times 10^{5}$ spores $\left./ \mathrm{ml}\right)$ were taken in small centrifuge tubes and then exposed to 20,40 , 60, 80 and 100 Gray of gamma absorbed doses from a $\mathrm{Co}^{60}$ as gamma source (GC 1200, BRIT). The dose range was selected on the basis of previous references [11-12,16].Then these irradiated suspensions were being serially diluted $\left(10^{-3}\right)$ and $300 \mu$ of diluted suspensions were inoculated in agar plates containing different $\mathrm{Cd}$ concentrations. After $3^{\text {rd }}$ day to 14 days of irradiation colony numbers were counted. In each case six replicates were done.

\subsection{Estimation of metal accumulation and removal potential (from respective liquid media) of Apergillus sp.:}

After 14days of irradiation one $8 \mathrm{~mm}$ disk of Aspergillus sp. colony was transferred from petri plates to same $\mathrm{Cd}$ concentrated liquid medium with a sterilized cork borer and allowed for growth in an orbital shaker at $100 \mathrm{rpm}$ for 14days of incubation at $29^{\circ}$. Estimation of total $\mathrm{Cd}$ accumulation in fungal body and removal percentage from respective culture media were carried out following the method of Srivastava and Thakur [17] using Atomic Absorption Spectrophotometer (FI-HG-AAS Perkin Elmer Analyst 400).

\section{RESULTS AND DISCUSSION}

Our results depict that gamma irradiation induced increase in Cd tolerance in Aspergillus sp.(grown under Cd stress) as reflected in increase in colony forming units (CFU) in gamma exposed groups in comparison to that of the unirradiated counterparts. Exposure to gamma (20-100Gy absorbed dose) could also effectively increase $\mathrm{Cd}$ accumulation and removal efficiencies of the fungi. Analysis of $\mathrm{Cd}$ tolerance in case of unirradiated Aspergillus $s p$ isolated from the waste dumping site showed that $90 \mathrm{ppm}$ of Cd completely inhibited growth of the fungi in lab condition, i.e. MIC of Cd is 90ppm. Soil of the site contained 3.6ppm of Cd.

Fig-1 reflects change in number of colony forming units (CFU) of Aspergillus $s p$ in response to different absorbed doses of gamma when grown under different concentrations of $\mathrm{Cd}$ in the medium. The pattern of change in CFU is different in case of different concentration of $\mathrm{Cd}$ supplemented in the medium. While gamma exposed Aspergillus sp. grown in 70ppm Cd in the medium showed gradual increase in CFU upto $60 \mathrm{~Gy}$ of gamma irradiation followed by decline in number of CFU (not below that of unirradiated but 70ppm $\mathrm{Cd}$ stressed group). 85ppm of $\mathrm{Cd}$ in the medium manifest a sharp increase in CFU from 20-40Gy (40Gy being maximum).In contrast 100ppm $\mathrm{Cd}$ supplementation which was not at all tolerated by Aspergillus $s p$ in unirradiated condition ( MIC-90ppm) showed maximum number of CFU in group irradiated at 20Gy of gamma followed by gradual decline in CFU in the groups exposed to other absorbed doses of gamma(Fig-1C] (as without gamma exposure Aspergillus $s p$ could not tolerate 100ppm of $\mathrm{Cd}$ so it is not comparable with that of unirradiated counterparts).

While irradiation at 60Gy of absorbed dose showed a 3 fold increase in CFU in Aspergillus sp grown in 70ppm Cd supplemented medium (Fig1A), a five-fold increase in CFU was observed in case of the group irradiated at $40 \mathrm{~Gy}$ absorbed dose of gamma and grown in $85 \mathrm{ppm}$ of $\mathrm{Cd}$ in the medium (Fig-1B), compared to that of the unirradiated but Cd exposed counterparts .

Similar to our results Dadachova et al.,[18] reported higher CFUs with increased biomass of some fungal strains isolated from areas having higher radiation level . Reports from some earlier researchers 
demonstrate stimulation of spore germination and growth of fungi exposed to low doses of gamma rays [1920].

Atomic Absorption Spectrophotometric (AAS) analyses reveal increase in Cd concentration in the fungal body together with decrease in $\mathrm{Cd}$ level in the growth media of the gamma irradiated groups of Aspergillus $s p$. This reflects that gamma exposed Aspergillus $s p$ showed better $\mathrm{Cd}$ accumulation and removal potential than their unirradiated counterparts (Fig-2). The most effective dose which reflected maximum CFU in all the three sets of fungal groups grown with three different concentrations of $\mathrm{Cd}$ in the medium following prior irradiation was considered for studying further accumulation and removal efficacies. A 60Gy exposed Aspergillus $s p$ could accumulate 1.73 times more $\mathrm{Cd}$ and could remove $11 \%$ more cadmium from $70 \mathrm{ppm} \mathrm{Cd}$ supplemented media with respect to its unirradiated counterpart. From $85 \mathrm{ppm}$ Cd supplimented media 40Gy exposed Aspergillus sp showed 1.65 times more accumulation and $10.5 \%$ more removal with respect to unirradiated counterparts. Without being exposed to gamma irradiation Aspergillus $s p$ could not tolererate $100 \mathrm{ppm} \mathrm{Cd}$ but after being irradiated, a 20 Gy exposed Aspergillus $s p$ could remove $45 \% \mathrm{Cd}$ from the $100 \mathrm{ppm}$ Cd supplimented media . Removal of Cd by Aspergillus niger was reported by Kumar et al. [21], who showed acclimated biomass of A.niger could remove $51.05 \% \mathrm{Cd}$ from liquid $\mathrm{Cd}$ supplemented media. A $43 \% \mathrm{Cd}$ biosorption efficiency of A.niger from single metal (Cd) liquid system has also been described earlier [22]. However. our study entailed an increase in removal efficiency of gamma irradiated Aspergillus sp. While unirradaited Aspergillus sp could show around $45 \%$ removal of $\mathrm{Cd}$ from the medium there was nearly $56 \% \mathrm{Cd}$ removal by the gamma irradiated groups. Thus it is evident that exposure to gamma triggers the metal tolerance of Aspergillus $s p$ and simultaneously induces augmentation in the uptake or accumulation potential and removal efficiency of the fungi. It may be postulated that gamma being a strong mutagenic agent, the concerned fungi subjected to gamma irradiation might have experienced mutation induction so as to become more tolerant towards Cd. No such reports are available so far where gamma has been used for enhancing metal tolerance in fungi and as such this is an effective report where gamma irradiation is being employed to enhance tolerance of fungi towards $\mathrm{Cd}$. Mutation of fungi for making them metal resistant are usually done by chemical applications [23-24]. Some groups have worked on use of UV radiation to induce metal resistance in bacteria [25-26].It is already reported that exposure to gamma showed maximum potential to induce mutation in fungi (Metarhizium anisopliae) than that of UV or other chemical mutagens [14].Gamma exposed fungal strain improvement for different industrial as well as ecological purposes are also reported earliar [27-28]. Thus our study showed that possibility of utilising gamma irradiation for increasing $\mathrm{Cd}$ removal efficiency of fungi.

\section{CONCLUSION}

This study is an effective report that might lead a new step forward towards Cd bioremediation. Utilisation of low doses of gamma irradiation for developing microbes with higher metal tolerance might possibly show the way us to formulate better approach for abatement of heavy metal pollution.

\section{ACKNOWLEDGEMENT}

Authors are very much thankful to UGC-DAE Consortium for Scientific Research, ,Kolkata centre, for giving gamma radiation facility as well as financial support to carry on this study and Department of Environmental Science, Kalyani University, for giving AAS facility.

\section{REFERENCES}

[1] G.M.Gadd. Interactions of fungi with toxic metals. The New Phytology.124,1993. 25-60.

[2] J.L.Wang, C.Chen . Biosorption of heavy metals by Saccharomyces cerevisiae: a review. Biotechnology Advances. 24.2006. 427451

[3] T.K. Pal, S.Bhattacharya,A.Basumajumdar. Cellular distribution of bioaccumulated toxic heavy metals in Aspergillus niger and Rhizopus arrhizus. International Journal of Pharma and Biosciences. VI (2),2010. 1-6

[4] E.Luef, T.Prey, C.P.Kubicek. Biosorption of zinc by fungal mycelial wastes. Applied Microbiology and Biotechnology. $34,1991$. 688-692.

[5] M.D.Mullen, D.C.Wolf, T.J.Beveridge, G.W.Bailey. Sorption of heavy metals by the soil fungi Aspergillus niger and Mucor rouxii. Soil Biology and Biochemistry. 24,1992. 129-135

[6] M.N.Akthar, P.M.Mohan. Bioremediation of toxic metal ions from polluted lake waters and industrial effluents by fungal biosorbent. Current Science. 69,1995. 1028 1030 .

[7] P.Bosshard, R.Bachofen, H.Brandl. Metal leaching of fly ash from municipal waste incineration by Aspergillus niger. Environmental Science and Technology. 30,1996. 3066-3070

[8] H.Jun,T. Kieselbach ,L.J. Jönsson . Enzyme production by filamentous fungi: analysis of the secretome of Trichoderma reesei grown on unconventional carbon source. Microbial Cell Factories. 2011 Aug 23;10:68. doi: 10.1186/1475-2859-10-68

[9] A.Anastasi , B.Parato, F.Spina, V.Tigini V., Prigione , G.C.Varese . Decolourisation and detoxification in the fungal treatment of textile wastewaters from dyeing processes. New Biotechnology..29(1). 2011.38-45.

[10] D.Park,Y.S.Yun, J.M.Park, Use of dead fungal biomass for detoxification of hexavalent chromium: screening and kinetics.Proces s Biochemistry.40.2005.2559-2565.

[11] S.I.N.Geweely , L.S.Nawar. Sensitivity to Gamma Irradiation of Post-harvest Pathogens of Pear. International journal of agriculture \& biology. 8(6),2006.710-716 
[12] T.Iftikhar, M.Mubashirniaz, Q.S.Abbas, M.A.Zia, I.Ashraf, K.J.Lee, U.I.Haq. Mutation induced enhanced biosynthesis of lipases by Rhizopus oligosporus var microsporus. Pakistan Journal of Botany. 42(2),2010. 1235-1249.

[13] R.C.Dubey, D.K.Maheswari. Practical Microbiology (First Edition . S.Chand and Company Ltd.,New Delhi,India Reprint ,2005).

[14] V.K.Cordeiro, E.A. Luna, J.L.Azevedo. Survival and mutant production induced by mutagenic agents in Metarhizium anisopliae. Science and Agriculture. 52(3), 1995.548-554.

[15] C.Tanaka, Y.Kubo, M.Tsuda .Comparison of mutagens in Cochliobolus heterostrophus mutagenesis. Annals of the Phytopathological Society of Japan. 54, 1988. 503-509

[16] M.Fadel , A.I.E. Batal. Studies on activation of amylolytic enzymes production by gamma irradiated Aspergillus niger using some surfactants and natural oils under solid state fermentation . Pak. J. Biol. Sci. 3(10),2000. 1762-1768

[17] S.Srivastava, I.Thakur . Biosorption Potency of Aspergillus niger for Removal of Chromium (VI).Current Microbiology. 53.2006. 232-237.

[18] E.Dadachova, R.A.Bryan. X.Huang, T.Moadel, A.D.Schweitzer, P. Aisen, J.D.Nosanchuk, and A.Casadevall. "Ionizing Radiation Changes the Electronic Properties of Melanin and Enhances the Growth of Melanized Fungi." PLoS ONE.2007. 2:e457

[19] A.M.Salama, M.I. Ali, Z.M. El-krdassy and T.M. Abdel-Rahman, Study on fungal radio resistance and sensitivity. Zbl. Bak. Abt. 132(II).1977. 1-13

[20] T.Tugay, N.N. Zhdanova, V.Zheltonozhsky, L. Sadovnikov, J.Dighton . The influence of ionizing radiation on spore germination and emergent hyphal growth response reactions of microfungi. Mycologia. 98(4).2006.521-7.

[21] A.Kumar,B.S.Bisht, V.D.Joshi. 2010. Zinc and Cadmium removal by acclimated Aspergilus niger: Trained fungus for biosorption. International Journal of Environmental Science and Research, 1(1)2010. 27-30

[22] A. Shoaib, T. Badar, N. Aslam. Removal of pb(ii), cu(ii) and cd(ii) from aqueous solution by some Fungi and natural adsorbents in single and multiple metal systems. Pakistan Journal of Boany, 43(6).2011. 2997-3000,

[23] W.B.Levine and G.A.Marzluf. Isolation and characterizationof cadmium resistant mutants of Neurospora crassa; Canadian Journal of Microbiology. 35.1989.359-365

[24] S.K.Pali, S.S.Konar, A.Mukherjee, T.K.Das. Removal of cadmium ion by cadmium resistant mutant of Aspergillus niger from water. Proceedings of the 10th International Conference of Environmental Science and Technology, Greece, 5-7 September2007.

[25] W.X.Ling, Q.G.Zhou,D.Jian, K.Jian, L.Xing. Mutagenic breeding of silver-resistant Acidithiobacillus ferrooxidans and exploration of resistant mechanism. Transaction of Nonferrous Metals- Society of China. 17, 2007. 412-417.

[26] J.Dib, J.Motok, V.F.Zenoff, , O.Ordonez, M.E.Farias . Occurrence of resistance to Antibiotics, UV-B, and Arsenic in Bacteria Isolated from Extreme Environments in High-Altitude (Above 4400 m) Andean Wetlands. Current. Microbiology .56 .2008.510517.

[27] M.A.M.Abo-State, A.I. Hammad, M.Swelim, R.B.Gannam. Enhanced Production of Cellulase(S) By Aspergillus spp. Isolated From Agriculture Wastes by Solid State Fermentation. American-Eurasian Journal of Agriculture and Environmental Science. 8(4). 2010. $402-410$.

[28] H.A.L.Mohamed and W.M.Haggag. 2005.Biocontrol potential of salinity tolerant mutants of Trichoderma harzianum against Fusarium oxysporum causing tomato wilt disease. Arabian Journal of . Biotechnology, 8 (1).2005. 35-48.

\section{FIGURES}

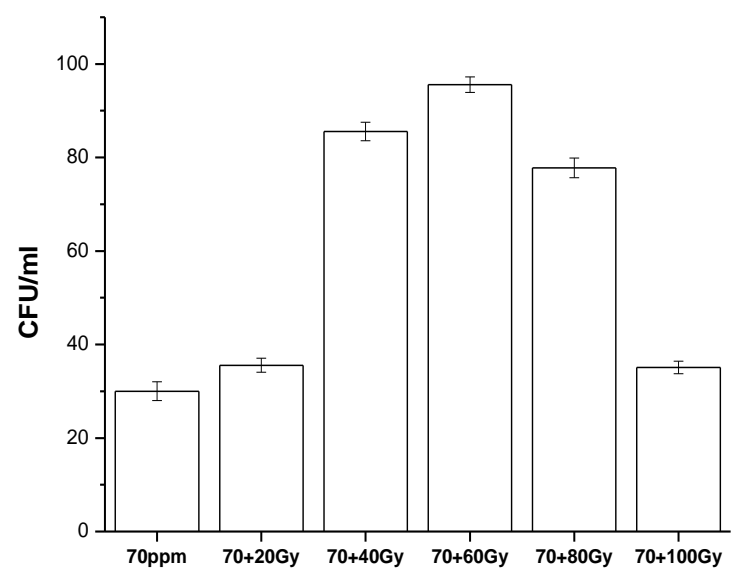

(A)

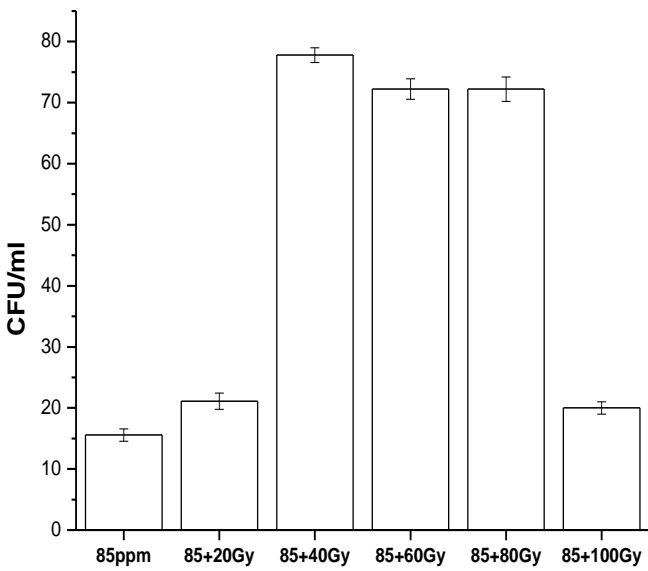

(B) 


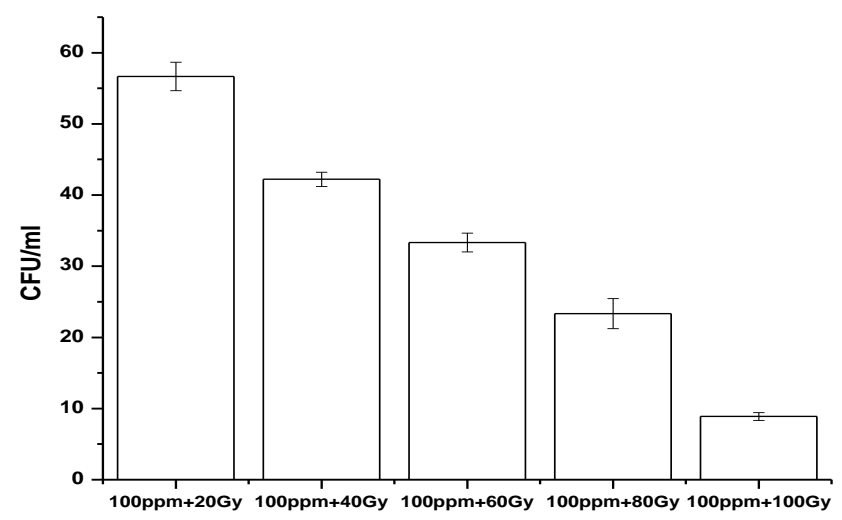

(C)

Fig-1 Colony forming unit (CFU) of irradiated Aspergillus sp with different doses of gamma irradiation (20-100Gy) and grown in medium supplimented with different concentrations of $\mathrm{Cd}$;

70ppm Cd(A), 85ppm Cd(B), 100ppm Cd (C)[n=6]

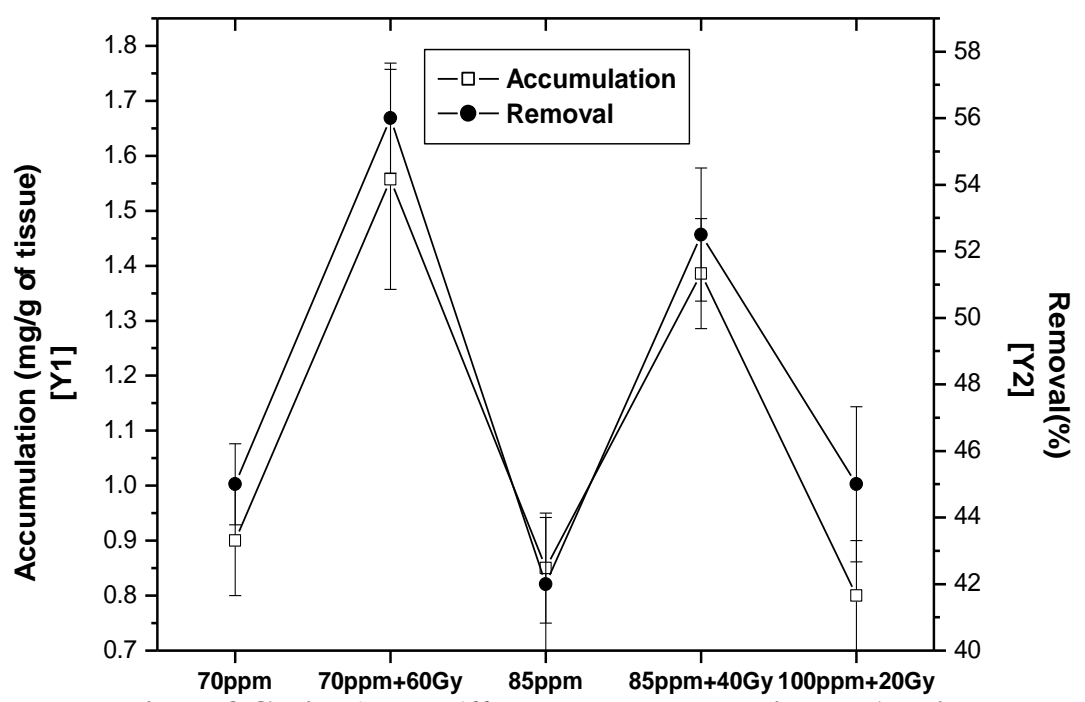

Fig-2 Bioaccumulation of $\mathrm{Cd}$ in Aspergillus $s p$ [plotted against $\mathrm{Y} 1$ axis] and removal of $\mathrm{Cd}$ by Aspergillus $s p$ [plotted against Y2 axis] exposed to different absorbed doses of gamma irradiation (20-100 Gy) and grown in media supplemented with different concentrations of $\mathrm{Cd}$; $[n=3]$ 